$63^{\text {ème }}$ Congrès de la SFCO, 03001 (2015)

DOI:10.1051/sfco/20156303001

(C) Owned by the authors, published by EDP Sciences, 2015

\title{
Lésions buccales induites par les thérapies ciblées inhibitrices de mTOR
}

\author{
Melka AC*, Makhoul R*, Devoize L*******, Collangettes D****, Bessard A*, \\ Deschaumes $\mathrm{C} * * * * * * *$ \\ * Service d'Odontologie, Département de Chirurgie Orale, Clermont-Ferrand \\ ** UFR d'Odontologie, Département de Chirurgie Orale, Clermont-Ferrand \\ *** INSERM/UdANeuroDol U1107, Clermont-Ferrand \\ **** Centre Jean Perrin, Clermont-Ferrand
}

Mots Clés : Mucite, thérapies ciblées, évérolimus, ulcérations buccales.

\section{Introduction}

Les traitements chimiothérapiques classiquement utlisés en cancérologie s'accompagent fréquemment de mucites et de difficultés d'alimentation, altérant ainsi l'état général des patients. Depuis quelques années, les limites des chimiothérapies cytotoxiques ont réorienté les recherches vers des biothérapies dites " ciblées » entrainant, elles aussi, des lésions buccales de type mucite. Parmi ces thérapies ciblées, on trouve les inhibiteurs de la protéine mammalian Target Of Rapamycine (mTOR), et notamment l'évérolimus. Ces thérapies provoquent le blocage de la glycolyse, une activation de l'apoptose ainsi qu'une anti-angiogenèse indirecte sur la tumeur visée.

\section{Observations}

Les mucites observées chez des patients traités par inhibiteurs de mTOR se manifestent après 5 à 25 jours de traitement (F. Martins et al. 2013). Elles apparaissent sous formes d'ulcérations aphtoïdes généralement au niveau de la muqueuse kératinisée, sans atteinte du tractus digestif. Contrairement aux ulcérations diffuses des chimiothérapies conventionnelles, elles sont généralement de petite taille, bien délimitées, possédant un fond fibrineux, des bords non surélevés et un halo érythémateux inconstant. L'atteinte est le plus souvent légère à miodérée avec $95 \%$ de grade 1 ou 2 (selon l'OMS). (A.L Watters et al. 2011).

Cependant, dans les cancers métastatiques, l'éverolimus peut être utilisé en association avec les chimiothérapies conventionnelles et engendrer des mucites au potentiel algique plus important et atteindre des grades élevés.

Conclusion

L'attitude thérapeutique face à ce nouveau type de mucite se résume à une prise en charge locale et symptomatique calquée sur la prise en charge des lésions chimio-induites. (M. de Oliveira et al. 2011). Néanmoins, cette attitude thérapeutique empirique reflète notre méconnaissance des mécanismes physiopathologiques précis de ces effets secondaires et nous incite à poursuivre les recherches en vue de pouvoir proposer des traitements préventifs et curatifs plus aboutis. 
$63^{\text {ème }}$ Congrès de la SFCO

Nom et adresse du conférencier

Anne-claire MELKA

Service d'Odontologie, Département de Chirurgie Orale

29 rue Blatin

63000 Clermont Ferrand (France)

anne-claire.melka@live.fr 